\title{
Governança da informação no contexto dos módulos contábeis dos sistemas ÉRP para a Indústria 4.0: Proposta de framework
}

\author{
Information governance in the context of ERPsystems accounting modules for Industry 4.0: a \\ framework proposal
}

\author{
Marcelo Demichelli Torres e Silva ${ }^{1}$, Rodrigo Baroni Carvalho ${ }^{2}$, José Márcio de Castro ${ }^{3}$, Marcos Vinícius Soares ${ }^{4}$ \\ 1 Pontifícia Universidade Católica de Minas Gerais (PUCMinas) e ICEG (Instituto de Ciências Gerenciais e Contábeis), MG, Brasil. ORCID: \\ https://orcid.org/0000-0001-5175-9592 \\ 2 Pontifícia Universidade Católica de Minas Gerais (PUCMinas), MG, Brasil. ORCID: https://orcid.org/0000-0003-3716-0879 \\ 3 Pontifícia Universidade Católica de Minas Gerais (PUCMinas), MG, Brasil. ORCID: https://orcid.org/0000-0002-3170-9456 \\ 4 Pontifícia Universidade Católica de Minas Gerais (PUCMinas), MG, Brasil. ORCID: https://orcid.org/0000-0002-4480-8744
}

Autor para correspondência/Mail to: Marcelo Demichelli Torres e Silva, marcelotdemicheli@gmail.com

Recebido/Submitted: 12 de junho de 2021; Aceito/Approved: 27 de agosto de 2021

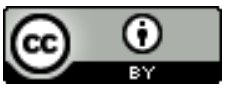

Copyright (c) 2021 Silva, Carvalho, Castro \& Soares. Todo o conteúdo da Revista (incluindo-se instruções, política editorial e modelos) está sob uma licença Cre-
ative Commons Atribuição 4.0 Internacional. Ao serem publicados por esta Revista, os artigos são de livre uso em ambientes educacionais, de pesquisa e não comerciais, com atribuição de autoria obrigatória. Mais informações em http://revistas.ufpr.br/atoz/about/submissions\#copyrightNotice.

\begin{abstract}
Resumo
Introdução: A gestão de dados e informações como ativos corporativos estratégicos se torna bastante desafiadora no contexto da Indústria 4.0, que traz consigo uma menor estruturação e maior volume de dados com o Big Data, bem como uma maior diversidade de fontes de dados com os sensores da Internet das Coisas. Problemas na Governança da Informação poderão resultar em fraudes, informações inconsistentes, comprometimento da tomada de decisões, roubos de dados e até mesmo multas, conforme previsto em legislação vigente. Ao longo de mais de duas décadas, os sistemas de gestão empresarial ERP (Enterprise Resource Planning) se consolidaram como pontos de convergência dos fluxos informacionais corporativos, sendo que seus Módulos Contábeis são um âmago desta integração e desempenham um papel significativo na Governança da Informação. Método: A base teórica desta pesquisa compreende os temas da Governança Corporativa, Governança da Tecnologia da Informação e Governança da Informação. A revisão de literatura considera a necessidade de adaptação dos sistemas ERP aos crescentes avanços tecnológicos da Indústria 4.0, notadamente em Big Data e Internet das Coisas. Resultados: Este trabalho, de cunho teórico, formula um novo conceito para Governança de Informação e propõe um framework para lidar com seus desafios no âmbito da Indústria 4.0. Conclusão: O trabalho discute cenários de aplicação do framework por gestores e profissionais de TI e controladoria em organizações cuja arquitetura informacional é centrada em sistemas ERP.
\end{abstract}

Palavras-chave: Governança da Informação; Sistemas ERP; Indústria 4.0; Contabilidade; Big Data; Internet das Coisas.

\begin{abstract}
Introduction: The electronic health record constitutes a support base for technological advances in the Health 4.0 area, an expression alluding to the Fourth Industrial Revolution or Industry 4.0. The innovations in this area include the use of robotics, artificial intelligence and connectivity to provide services to the patient. This research was presupposed on the fact that the use of archivistics methods and Blockchain and Smart Contracts technologies can contribute positively to the management of the patient's electronic health records. The aspects covered by this management are defined in the production, use and destination of health records as well as the protection, security and privacy of your data. Method: The systematic search of the literature and studies of the works in the sub-area "Archivology" were the methods used to identify and describe as activities of an archivistics management, the attributes of the technologies Blockchain and Smart Contracts and the relationship between these themes with the governance of health records. The research had an exploratory, descriptive, qualitative character and a constructivist ontological perspective. Result: The studies contributed to finding gaps in the legislation and in the production of scientific articles on the subject investigated, as well as to identify possible technological solutions to problem-solving of technical issues that had not yet been clarified. Conclusion: The participation of archivists in research related to archivistics management associated with Blockchain and Smart Contracts technologies is necessary to contribute to the evolution of record management, of which the patient's electronic health records are part.
\end{abstract}

Keywords: Eletronic health records; Archivistics; Blockchain; Smart Contracts; Health 4.0.

\section{INTRODUÇÃO}

A partir da década de 1990, as organizações empreenderam esforços para integrar toda a produção e gestão fabril com os sistemas de gestão empresarial ERP (Enterprise Resource Planning), que constituem a matriz tecnológica principal das ferramentas de gestão das organizações (Hasan, Ebrahim, Mahmood, \& Rahman, 2018). Os sistemas ERP têm como premissa básica fazer a comunicação de todas as áreas da empresa de forma integrada, possibilitando a transferência e permuta de informações por todos os seus setores. A integração tecnológica promovida pelos sistemas ERP, tendo a Contabilidade como ponto de convergência, constituiu relevante conquista corporativa para permitir um melhor fluxo informacional entre os setores da empresa bem como ao longo da cadeia de valor (Dani \& Beuren, 2018). 
Apesar da evolução dos sistemas de ERP, o movimento de inovação tecnológica denominado Indústria 4.0 pode ter implicações para estes sistemas. Também chamada de quarta revolução industrial (Schwab, 2019), trata-se de uma nova abordagem da maneira de produção nas organizações, a qual traz mudanças e implicações nos processos de fabricação (B. P. Santos, Alberto, Lima, \& Charrua-Santos, 2018). Em conjunto com as diversas soluções e propostas inovadoras que surgem no mercado, é relevante tratar como elas estarão interligadas com o ERP e como será a Governança da Informação destas bases, considerando a necessidade de uma aderência sólida e eficiente aos atuais sistemas integrados (Simões, Rodrigues, Maccari, \& Pereira, 2011).

O tema Governança da Informação (GI) está inserido no contexto mais amplo da Governança Corporativa (GC), que ganhou grande proporção no início dos anos 2000, quando se passou a exigir das organizações um conjunto de mecanismos de governança para que as empresas se tor-nassem mais transparentes (Andrade \& Rossetti, 2009). O tema da Governança da Informação no contexto da Indústria 4.0 e sua interface com um sistema integrado de gestão (ERP) precisam ser observados e pesquisados mais amiúde, visto a relevância da contribuição da pesquisa para os teóricos e praticantes (Medeiros, Lavarda, \& Erdmann, 2020). Partindo da percepção de que dados e informações são ativos corporativos estratégicos, a motivação deste trabalho se concentra na gestão destes ativos, a qual se torna bastante desafiadora no contexto de legislações mais rigorosas sobre o uso de dados, tais como a LGPD (Lei Geral de Proteção de Dados) no âmbito nacional, e também da Indústria 4.0 e, em particular, do Big Data, que traz consigo questões sobre o volume, variedade, velocidade e veracidade dos dados (4Vs).

Problemas na Governança da Informação poderão resultar em fraudes, informações inconsistentes, comprometimento da tomada de decisões, roubos de dados e até mesmo multas e processos judiciais, conforme previsto em legislação vigente (Furlan \& Laurindo, 2019). Há, no campo organizacional, uma negligência em relação aos fatores que afetam o ambiente informacional e uma literatura incipiente sobre Governança da Informação (Maçada, Brinkhues, Freitas Jr, \& Lajara, 2019). Com o surgimento de novas tecnologias e o rápido crescimento das redes de informação em todo o mundo, as empresas precisam ampliar cada vez mais suas medidas de estruturação de governança de dados e informação (Li et al., 2019). A combinação dos elementos supracitados constitui a lacuna teórica e a motivação empírica para o desenvolvimento deste trabalho.

Entre as diversas áreas empresariais, pode-se destacar a contabilidade como um ponto nevrálgico para a Governança da Informação centrada em sistemas ERP. As informações geradas na Contabilidade das empresas, que antes eram relatórios utilizados por poucos agentes, como auditorias, bancos e fiscais, estão cada vez mais sendo demandadas por outros segmentos da sociedade, sejam empresas, investidores, financiadores, estudantes e pesquisadores (Dani \& Beuren, 2018). Entende-se que os módulos contábeis são o cerne deste grande legado informacional que é o sistema de gestão integrada (ERP). Tal legado é fruto de décadas de investimentos corporativos em parametrizações, customizações, atualizações de versões e treinamento de usuários. Este legado precisa ser preservado e adaptado ao movimento da Indústria 4.0, que trará inovações como dados sendo fornecidos não apenas por seres humanos de maneira estruturada, mas também por dispositivos IoT (Internet of Things) e em formatos não tão estruturados no âmbito do Big Data. Portanto, o ERP deve ser considerado como uma variável relevante para a equação da Indústria 4.0.

A Governança da Informação (GI) vem se tornando cada vez mais importante para gestores e contadores ganharem entendimento sobre como transformar o ambiente de Big Data em informações úteis e governáveis (Coyne, Coyne, \& Walker, 2018). Há espaço para ampliação e criação de melhorias no campo da GI, com demanda para novas pesquisas e novos modelos de maturidade (Proença, Vieira, \& Borbinha, 2016). Face à crescente complexidade, são necessárias mais pesquisas sobre o impacto da governança associado ao crescimento tecnológico nas organizações em várias categorias: Clientes, Estratégia, Sistemas de Informação (grifo nosso), Qualidade, Desempenho, Colaboradores, Riscos, Orçamento e Custos (Carraro, Meneses, \& Brito, 2019).

Considerando a lacuna teórica evidenciada pelos autores supracitados, o objetivo deste artigo é articular um conceito atualizado para Governança de Informação e propor um framework que relacione as categorias e práticas da Governança da Informação com o contexto dos módulos contábeis do ERP no âmbito da Indústria 4.0. A seção seguinte descreve os passos realizados para a construção do referencial que norteou o desenvolvimento do framework. O referencial teórico contempla as temáticas da Indústria 4.0, sistemas ERP, Big Data, governança corporativa, maturidade e governança da informação. Na sequência, o framework e o conceito de Governança da Informação são apresentados. A última seção discute as possibilidades de aplicação do framework em trabalhos futuros bem como as suas limitações.

\section{METODOLOGIA DA REVISÃO DA LITERATURA}

Elaborar um bom roteiro da revisão de literatura é importante para atingir os objetivos da pesquisa, gerar segurança ao estudo e ampliar a obtenção de resultados (Neupane, 2020). Assim sendo, este contemplou duas etapas temáticas. A primeira compreendeu as características dos sistemas ERP, sua governança e sua evolução, o ERP e sua integração ao cenário de Indústria 4.0 com ênfase em IoT e Big Data.

Por sua vez, a segunda etapa buscou entender como se estrutura a Governança da Informação centrada em Sistemas de Gestão Empresarial ERP com ênfase nos módulos contábeis. A busca de literatura se iniciou 
com o tema da Governança Corporativa de maneira abrangente para, em seguida, focar a busca nos temas de Governança da Tecnologia da Informação, Maturidade da Informação e Governança da Informação no contexto da Indústria 4.0. A revisão acerca do tema de pesquisa colabora para se avaliar com melhor critério o conteúdo, delimitando a unidade de leitura, auxiliando o pesquisador na captação de fontes de ideias para novas investigações e orientando-o em relação ao que já é conhecido (Echer, 2001), sendo este o caso deste trabalho, o qual se propõe a analisar um recorte da literatura existente para, em seguida, formular um novo conceito de Governança da Informação bem como um framework no contexto da integração dos módulos contábeis do ERP ao universo da Indústria 4.0 .

A busca por documentos para revisão de literatura foi efetuada a partir de combinações dos seguintes termos: Indústria 4.0, sistemas ERP, Big Data, Governança Corporativa, Governança de Tecnologia da Informação e Governança da Informação. Foram utilizadas as seguintes bases de dados: portal Capes, Scielo, IEEE (Institute of Eletrical and Electronic Engineers) e SPELL (Scientific Periodicals Electronic Library). A pesquisa foi realizada em outubro de 2020 e resultou em 134 artigos, os quais foram avaliados, organizados e unificados em uma base de dados. Após aplicar os critérios para avaliar os documentos recuperados, foram selecionados 84 documentos estratificados pela temática central segundo a Tabela 1.

\begin{tabular}{lr}
\hline Assuntos & Números de Artigos \\
\hline Governança da Informação & 45 \\
\hline Indústria 4.0 (Big Data / Internet das Coisas) & 16 \\
\hline Governança Corporativa & 12 \\
\hline ERP & 9 \\
\hline Governança Contábil & 2 \\
\hline Total de Artigos & $\mathbf{8 4}$ \\
\hline
\end{tabular}

Tabela 1. Temática Central dos Artigos Selecionados Fonte: dados da pesquisa.

A leitura e fichamento dos artigos selecionados priorizaram a identificação de categorias e métricas associadas à Governança da Informação, emergindo os aspectos da maturidade da informação, integridade, confiabilidade, rastreabilidade da informação, qualidade, tempestividade e segurança. Tal categorização foi fundamental para construir os esboços que resultaram na proposição do framework que almeja responder à seguinte questão: "Como se estrutura a Governança da Informação centrada em Sistemas de Gestão Empresarial ERP no contexto da Indústria 4.0? A seção seguinte detalha o referencial teórico empregado na construção do framework.

\section{ANÁLISE DO REFERENCIAL TEÓRICO}

A abordagem do referencial teórico se sustenta na arquitetura de informação para Indústria 4.0, os sistemas ERP, o ERP \& Indústria 4.0, Big Data, governança corporativa e Governança da Informação, assunto principal deste artigo. Enfoca-se também a função da Contabilidade, que é o recorte teórico e empírico para análise do ERP neste contexto; e a governança da utilização de soluções de tecnologias e sua integração com o sistema de gestão.

\section{ERP no Contexto da Indústria 4.0}

Em 2011, a Alemanha lançou um projeto denominado "Industrie 4.0" que promoveu o início da transformação digital da manufatura e alavancou novas tecnologias. Os principais pilares da Indústria 4.0 são: Internet das Coisas (Internet of Things - IoT), realidade aumentada, robôs autônomos, simulação, manufatura aditiva, Big Data, tecnologia em nuvem (cloud computing), segurança cibernética e integração horizontal e vertical de sistemas e softwares (S. Santos, 2018).

A participação do sistema ERP é essencial neste processo de convergência, pois a sua finalidade é servir como a instância central para dados mestre e dados transacionais em todos os negócios relacionados com processos nas empresas (Araújo, Lima, \& Sobral, 2018). Com a ajuda de interfaces, os sistemas ERP serão conectados com dispositivos de entrada e saída, como scanners, dispositivos de ilustração e comunicação, bem como outros sistemas de software e hardware dentro e entre empresas. Consequentemente, o sistema ERP pode ser visto como uma parte essencial da rede de sistemas ciber-físicos (CPS - Cyber Physical Systems), portanto, precisa ser levado em consideração (Telukdarie, Buhulaiga, Bag, Gupta, \& Luo, 2018).

No entanto, um dos maiores desafios está em integrar os sistemas atuais da companhia com a Indústria 4.0 (Morais, Moura, \& Denani, 2018). O recorte desta pesquisa no amplo contexto da Indústria 4.0 é a aplicação da Internet das Coisas (IoT) ao processo industrial, bem como do Big Data, ambos como aspectos que precisam estar no âmbito da Governança da Informação. Big Data é um termo que traz em seu significado a noção do seu tamanho (Sharma, 2017), sendo a sua governança imprescindível para as organizações, conforme discutido a seguir. 


\section{Big Data e Internet das Coisas (IoT)}

O crescimento exponencial do volume de dados permite às organizações armazenar amplas bases de dados, porém é necessário que tais ativos criem valor para as organizações. Big Data pode ser definido como um termo que compreende todos os aspectos envolvidos na geração, aquisição, transmissão, armazenamento, processamento, transformação, análise e apresentação de dados para qualquer finalidade (Pinto, Cardoso, Pinheiro, Parreiras, 2019). ? destaca três categorias de desafios para o Big Data: os desafios dos dados, dos processos e da gestão (Figura 1).

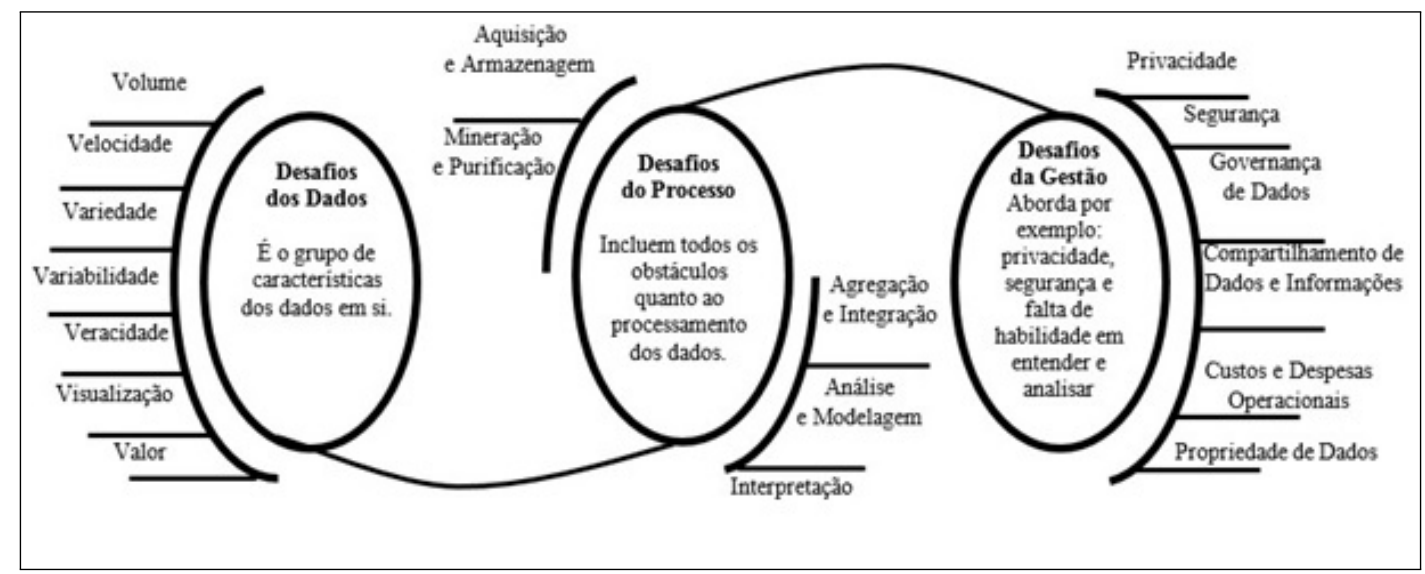

Figura 1. Classificação conceitual dos desafios do Big Data. Fonte: Sharma (2017).

A solução de Big Data passa por uma governança de dados cada vez maior e mais complexa, considerando pelo menos o volume, a velocidade, a variedade, veracidade e o valor dos dados, entendido como o quinto $\mathrm{V}$ por Sharma (2017). Volume significa a quantidade acumulada de dados e seu fluxo de entrada. Velocidade representa o ritmo de dados usados para interações e variedade significa a quantidade de formatos e estruturas de dados (Pinto et al., 2019). A IBM acrescentou outro "V" para a dimensão da veracidade em resposta às questões de qualidade e de origem de seus dados (Sharma, 2017). A Microsoft ampliou essa classificação, incluindo o conceito de Visibilidade, enfatizando o que é exigido para ter uma imagem completa dos dados, a fim de tomar uma decisão informada (Pinto, Cardoso, Pinheiro, \& Parreiras, 2019). Posteriormente, (Sharma, 2017) acrescentou outros três "V": o veredicto, que é uma escolha potencial ou decisão que deve ser feita com base no escopo do problema e recursos disponíveis; a validade, que enfatiza como adquirir corretamente os dados e, por último, a variabilidade, que é como usar o processo de inferência lógica para reduzir a complexidade de dados e chegar a resultados ou previsões desejáveis para as necessidades de negócios. A Figura 2 representa uma síntese das visões encontradas sobre as dimensões de Big Data.

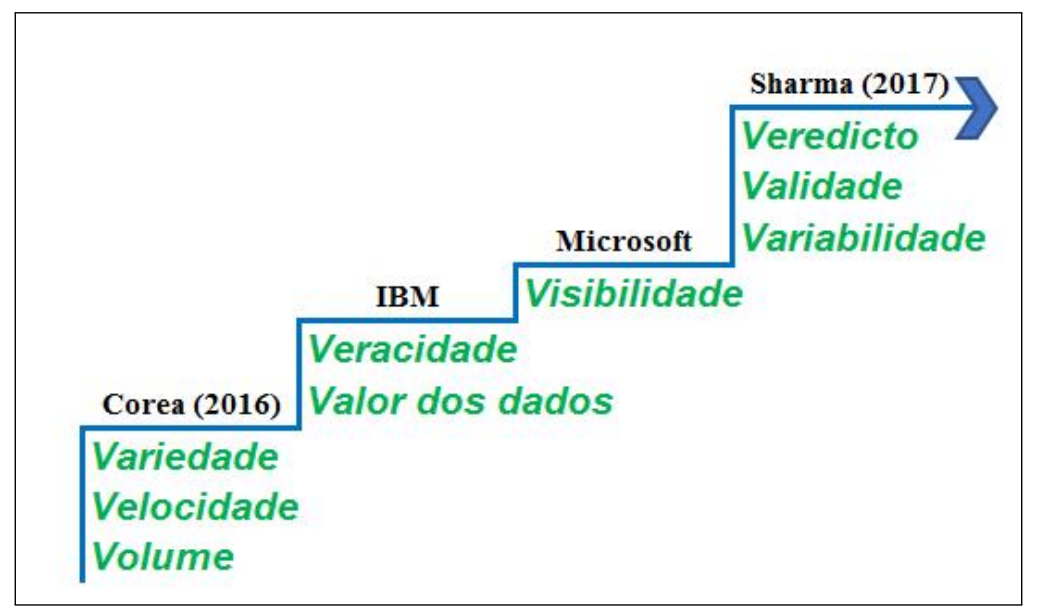

Figura 2. Evolução de Big Data e suas dimensões .

Fonte: os autores.

As organizações usualmente armazenam grandes volumes em suas bases sem saber como tratar tantos dados (Hurwitz, Kaufman, \& Bowles, 2015). Considerando dados como ativos, as empresas estão constantemente buscando maneiras de viabilizar economicamente o tratamento do valor crescente de dados, sendo que a forte reputação corporativa está usualmente associada às boas práticas de governança (Dutra, Parente, Dutra, \& Parente, 2018). Neste contexto, o Big Data é tratado pelas empresas como um processo relevante dentro de 
um contexto de melhorar e aproveitar as competências e capacidades operacionais e estratégicas, influenciando positivamente o desempenho financeiro corporativo (Pinto et al., 2019).

Há muitos desafios na utilização do Big Data, entre eles sua governança (Akerkar, 2014). O desafio no processo de governança de um grande banco de dados é organizar por categorias, modelar as demandas da organização, mapear os dados à medida que são capturados e armazenados, principalmente devido à natureza não estruturada e complexa dos dados. Este procedimento é imprescindível para assegurar a qualidade dos dados extraídos e analisados (Hashem et al., 2015). As práticas de contabilidade, principalmente orçamento, custeio e avaliação de desempenho das organizações, serão influenciadas e modificadas por causa do Big Data (Gärtner \& Hiebl, 2018), demandando uma participação mais efetiva da Contabilidade na Governança da Informação (Coyne et al., 2018).

Por sua vez, a Internet das Coisas (IoT - Internet of Things) consiste em sensores, dispositivos e atuadores middleware para coletar e armazenar dados de modo integrado com aplicativos e ferramentas de análises para apoiar e até mesmo realizar ações autônomas (International Business Machines [IBM], 2019). A combinação de dados gerados por meio da Internet das Coisas ajuda a reconhecer padrões e prever falhas ou problemas de qualidade do produto antes que aconteçam. A maioria das fábricas é composta por ativos de tecnologia de operação como máquinas, linhas de equipamentos e dispositivos robóticos que nem sempre estão conectados. A tendência está se inclinando para uma manufatura inteligente com um chão de fábrica mais conectado. Neste novo ambiente industrial, atributos como conectividade, Inteligência Artificial, Ciência de Dados, Big Data, IoT, aprendizagem de máquina entre outros irão mudar o fluxo e quantidade de dados existentes nas empresa (B. P. Santos et al., 2018). Assim, torna-se necessário adaptar e ampliar a Governança de Informação.

\section{Governança Corporativa}

Para se compreender a Governança da Informação, é importante entender o contexto da estrutura de governança de uma organização. Governança corporativa seria o primeiro nível de gestão de uma organização, caracterizada pelos controles e procedimentos que existem para assegurar que a administração sempre atenda ao seu público de interesse (Kanagaretnam, Lobo, \& Whalen, 2007).

Nos anos 90, a governança corporativa se intensificou nas empresas e comunidades de negócios e finanças (Meirelles, 2005). Na busca de ferramentas que auxiliassem no crescimento, no acerto das decisões e na maximização dos resultados, a Governança Corporativa emerge como uma ferramenta capaz de manter o equilíbrio organizacional, formulando um eficiente conjunto de mecanismos, tanto de incentivos quanto de monitoramento, adaptado à realidade de cada entidade empresarial como forma de assegurar que o comportamento dos administradores esteja sempre alinhado com os interesses da organização e com objetivo de recuperar, manter e garantir a confiabilidade da entidade contábil perante os seus usuários, sejam eles fornecedores, credores, acionistas e afins (Lazzarotto, 2015).

Por sua vez, o mercado financeiro vem exigindo das empresas maior transparência, governança e confiabilidade nas informações e demonstrações contábeis, garantindo credibilidade aos resultados apresentados. A governança corporativa auxilia acionistas, sócios e administradores na tomada de decisão de seus negócios, trazendo equilíbrio e respeito quanto aos interesses entre o mercado e seus objetivos empresariais (Souza, Bauer, \& Coletti, 2020).

Inserida na estrutura de gestão da organização, além da governança corporativa, encontram-se dois construtos distintos: a Governança da Informação (GI), objeto principal desta pesquisa, e Governança da Tecnologia da Informação (GTI) (Teodoro, Przeybilovicz, \& Cunha, 2014). A GTI se concentra nos processos, nas estruturas e nas interfaces que criam sinergia em prol do alinhamento entre a TI (Tecnologia da Informação) e áreas de negócio (Dinsmore \& Rocha, 2015). Um dos objetivos da GTI é abrir a "caixa-preta" dos investimentos em hardware, software e serviços de TI, envolvendo usuários e gestores na tomada de decisão sobre o uso destes recursos. Por seu turno, a Governança da Informação (GI), como será detalhada na sequência, surgiu como uma nova frente na área de governança corporativa cujo propósito é a produção e armazenamento dos dados, sendo considerada como um ativo importante na administração da companhia (Haes \& Van Grembergen, 2009).

\section{Governança da Informação}

A principal característica da GI é a visão da informação como um ativo crítico da empresa, informação esta que deve ser utilizada para detectar e agir sobre as oportunidades (Bruening \& Waterman, 2010), contribuindo para criar um cenário favorável de modo que as organizações e seus usuários possam atribuir significado aos seus dados (Beijer, Kooper, \& Advisory, 2010). A existência da informação assimétrica nas organizações traz prejuízo na qualidade percebida desta informação, que, com o passar do tempo, pode gerar perda de confiança. Tal contexto colabora para que as empresas criem instituições e mecanismos como a Governança da Informação para combater essa assimetria (Akerkar, 2014).

Governança da Informação refere-se ao estabelecimento de políticas, por meio de estruturas formais, que definem regras, procedimentos e direitos decisórios sobre a gestão da informação, os quais beneficiarão a instituição (Faria, Maçada, \& Kumar, 2012). A existência de políticas claras, procedimentos operacionais, padrões de configurações 
e documentação são relevantes (Gianella \& Gujer, 2006) e precisam ser avaliados, controlados e estruturados. A Governança da Informação estabelece um ambiente de oportunidades, regras e direitos decisórios para a valoração, criação, coleta, análise, distribuição, armazenamento, uso e controle de informações (Kooper, Maes, \& Lindgreen, 2011). A GI proporciona a ampliação de controles, processos e tecnologias desenhadas para auxiliar organizações a maximizar o valor dos ativos de informação, facilitando o alinhamento com o sistema de gestão empresarial ERP (Enterprise Resource Planning) utilizado pela administração (Blair \& Murphy, 2011).

Para o desenvolvimento deste trabalho, são associadas práticas de governança e suas categorias, entre elas: tempestividade, qualidade e segurança. A tempestividade busca garantir que as interfaces sejam feitas on-line, ou no momento mais adequado para compartilhamento e utilização da informação. A informação fica disponível para ser utilizada quando necessária, sendo que o tempo de resposta e ação será medido e controlado. Em outras palavras, a tempestividade de um sistema reflete a rapidez e o melhor momento demandado da informação para seus usuários (Dias, 2002). A tempestividade se refere à frequência e velocidade, ou seja, aos intervalos de tempo requeridos para a produção das informações e à defasagem de tempo entre quando uma informação é requerida e quando ela vai estar disponível (Bouwens \& Abernethy, 2000).

A qualidade exige que a informação seja íntegra, sem falhas e vieses, com confiabilidade da origem do dado, de forma a refletir com total integridade as movimentações e os processos iniciais. A governança e a qualidade dos dados e das informações têm sido discutidas no campo organizacional (Freitas, Reis, Michel, Gronovicz, \& de Macedo Rodrigues, 2013), tendo em vista o fato de a produção de dados transacionais crescer exponencialmente, ampliando sua complexidade e interconectividade. Alterações nas regras de negócio, estratégias de integração de negócios corporativos, fusões, novas aquisições e parcerias podem ocasionar misturas de fontes de dados por parte dos departamentos de TI e contabilidade, devendo-se redobrar a atenção e traçar estratégias e projetos capazes de absorver as mudanças e, ao mesmo tempo, atender às necessidades do negócio (Mangueira \& Alves, 2014). Presença de auditorias internas, conciliações de valores e análise de conteúdo são relevantes para a garantia da qualidade da informação, criando, assim, valor para a informação.

Já o conceito de segurança da informação está ligado à confidencialidade, integridade e disponibilidade da informação (Smallwood, Kahn, \& Murphy, 2014). Além de controlada, a informação deve estar associada a todas as plataformas existentes (Atkinson, Kaplan, Matsumura, \& Young, 2011), principalmente neste contexto de transformação tecnológica promovida pela Indústria 4.0, em que a integração é importante para garantir o fluxo informacional entre todos os processos existentes na companhia. O gerenciamento da segurança da informação engloba aspectos de gestão de riscos, de tecnologias da informação, de processos de negócios, de recursos humanos, da segurança física e patrimonial, de auditoria, de controle interno e de requisitos legais e jurídicos (Rigon \& Westphall, 2013). Estes atributos informacionais de tempestividade, qualidade e segurança estão também presentes, de modo crítico no contexto das informações contábeis, conforme será debatido a seguir.

\section{Governança das Informações Contábeis}

Para as empresas, as informações de caráter financeiro e de controle são consideradas umas das mais importantes de um sistema ERP, uma vez que atendem às necessidades de informação nos níveis estratégico e tático (Shkurskii \& Sabel'nikova, 2011). A contabilidade, uma área sensível ao crescimento e absorção de dados, tem uma forte capacidade de identificar as necessidades informacionais e de controles para tomadores de decisão internos e externos, desempenhando um papel significativo na Governança da Informação e no suporte empresarial.

Com base nos autores pesquisados e nos diversos conceitos sobre a GI, considerando a realidade da Indústria 4.0, este trabalho formula um novo conceito para o tema. Governança da Informação é a transformação do dado em informação, ativo de uso e subsídio para tomada de decisão, garantindo sua tempestividade, qualidade e segurança para todas as plataformas e ferramentas utilizadas pelos seus usuários, buscando criar valor para companhia. A integração entre os dados cada vez mais volumosos, reflexo de um cenário crescente de inovações industriais, bem como sua aderência ao ERP, demanda governança para transformar e criar valor a partir dos ativos informacionais da organização. A seção seguinte apresenta um framework que instrumentaliza este conceito.

\section{CONSTRUÇÃO DO FRAMEWORK}

O framework proposto (Figura 3) apresenta a dimensão da organização sendo influenciada pelos avanços vindos da Indústria 4.0. As entradas tecnológicas (inputs) são oriundas do Big Data e a Internet das Coisas e as saídas envolvem partes interessadas (stakeholders) como diretorias, gerências, investidores, usuários internos e externos. Neste contexto, aliado ao crescente volume de dados oriundos do Big Data, tem-se a adaptação aos sistemas ERP nas organizações.

Todas as informações empresariais, cadastro, ordens de serviços, produções, compras, vendas e contratos podem ser armazenadas com possibilidade de conectividade e interligação. Com o crescimento das informações 
disponíveis, deve ser adotado um modelo institucional de Governança da Informação que controle e oriente a empresa com qualidade, disponibilidade, facilidade de utilização, integridade, controle e segurança de informação.

Além dos padrões, políticas e processos bem definidos, o estabelecimento de avaliações da qualidade da informação se faz necessário. A qualidade da informação é uma categoria que pode transformar a informação em um ativo crítico da empresa (Zonnenshain \& Kenett, 2020). Outra categoria analisada é a Segurança da Informação.

A Governança da Informação fornece políticas e métricas para auxiliar na tomada de decisões e gerenciar a conformidade e os riscos em toda a organização. Além das categorias de Segurança e Qualidade, precisa-se medir e analisar a disponibilidade da informação. Em que momento do processo a informação estará disponível é um atributo relevante para o processo de governança do ambiente empresarial (Trindade, Oliveira, \& Becker, 2011). Além de ter a informação com qualidade e segurança, é necessário tê-la tempestivamente, atendendo as demandas empresariais.

A informação deve ser governada, baseada em evidências, monitorada, com elevados níveis de maturidade e integração, constituindo uma base substancial de apoio à quarta revolução industrial (Zonnenshain \& Kenett, 2020). Qualidade da Informação é percebida como uma informação que seja relevante para uso dos seus consumidores (Melkas \& Uotila, 1999), atendendo os seus diversos usuários e garantindo melhor tomadas de decisões de negócio. As estruturas de Governança da Informação englobam políticas, práticas e mecanismos que almejam ampliar os benefícios dos dados e das informações para a organização (Khatri \& Brown, 2010).

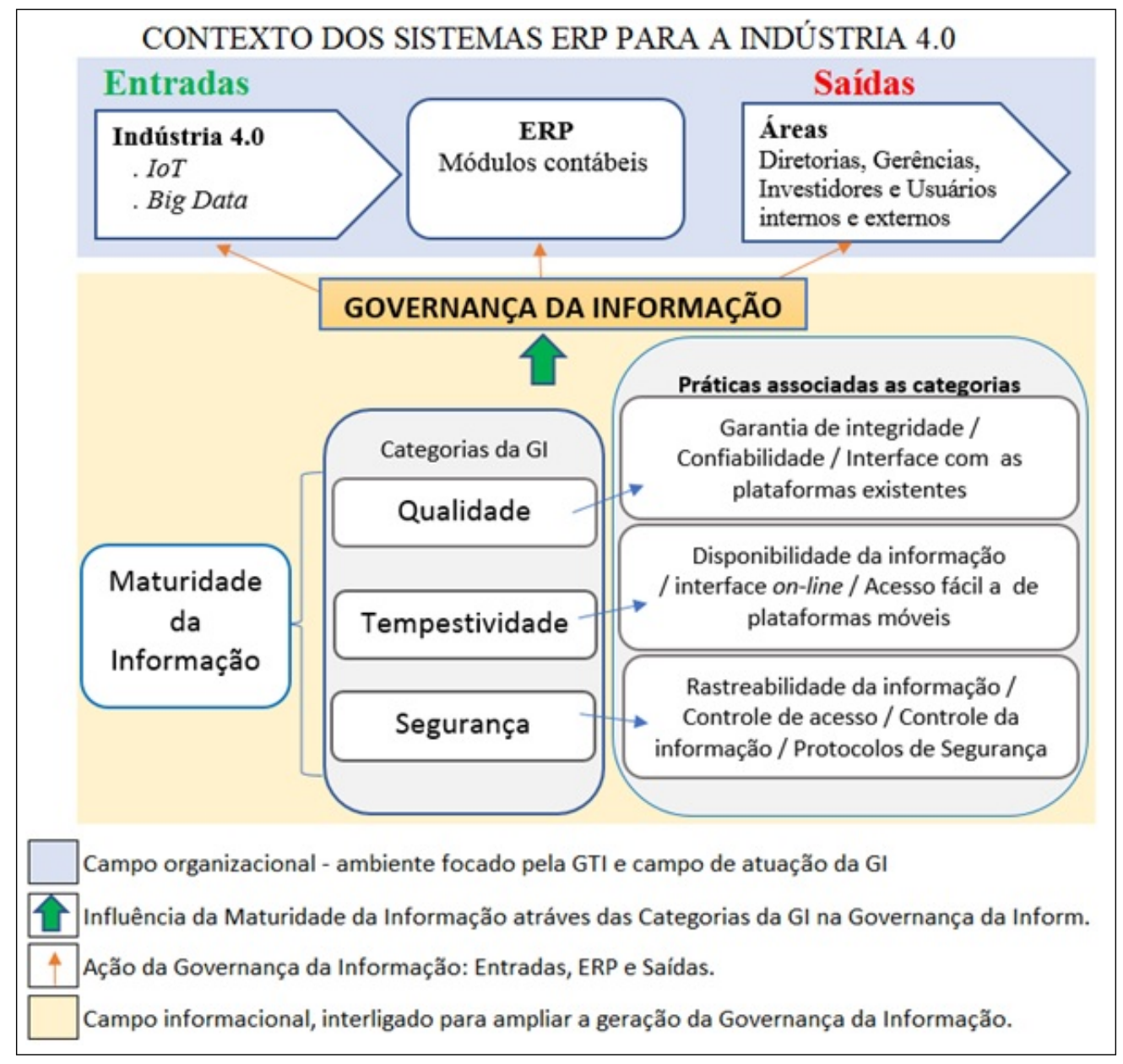

Figura 3. Governança da Informação - Contexto dos Sistemas ERP para a Indústria 4.0.

Fonte: os autores.

Com o crescimento das informações disponíveis, deve ser adotado um modelo institucional de Governança da Informação que controle e oriente a empresa com qualidade, disponibilidade, facilidade de utilização, integridade e segurança, garantindo a maturidade da informação.

A criação de protocolos de segurança e proteção contra perda, alteração ou divulgação indevida de dados permite à organização gerir riscos e orientar as ações de negócio. Um programa abrangente de controles, processos e tecnologias auxilia as organizações a maximizar o valor dos ativos de informação, enquanto minimiza riscos e custos. Segurança da informação é essencial para redução ou mitigação de risco, além de assegurar a eficiência operacional, alcançar e manter a vantagem competitiva da instituição. A Governança da Informação fornece políticas e métricas para auxiliar na tomada de decisões e gerenciar a conformidade e os riscos em toda a 
organização (Blair \& Murphy, 2011).

Além das categorias de Segurança e Qualidade, precisa-se medir e analisar a disponibilidade da informação. Em que momento do processo a informação estará disponível é um atributo relevante para o processo de governança do ambiente empresarial (Trindade et al., 2011). Além de ter a informação com qualidade e segurança, é necessário tê-la tempestivamente, atendendo as demandas empresariais.

\section{CONCLUSÕES}

A informação deve ser governada com elevados níveis de maturidade e integração, constituindo uma base substancial de apoio à Indústria 4.0 (Zonnenshain \& Kenett, 2020). Frameworks baseados em informação capturam dimensões importantes que auxiliam usuários e gestores no entendimento e atendimento das necessidades de qualidade da informação por parte de seus consumidores de produtos e serviços de informação (Presser \& da Silva, 2019).

O framework proposto neste trabalho busca atender tais requisitos ao trazer uma perspectiva atualizada da Governança da Informação face aos avanços da Indústria 4.0. Esta perspectiva preserva o ERP como sistema legado e reconhece seu papel consolidado na integração das informações corporativas, em particular as de natureza contábil. Convém ressaltar a crescente necessidade por controle de dados face à LGPD (Lei Geral de Proteção de Dados) e o elevado grau de utilização da Internet, o que representa riscos e dados de natureza não estruturada. Neste contexto, atributos como Qualidade, Tempestividade e Segurança da Informação devem continuar a ser observados pelos gestores e usuários da informação.

O framework aqui descrito evidencia a necessidade de verificação de integridade das informações advindas do Big Data e da Internet das Coisas, mitigando perda de informação ou distorções que possam atingir de forma sensível as bases de dados contábeis. Outro ponto relevante é a tempestividade associada ao tempo, ao momento de produção e à disponibilidade do dado para que ele possa ser devidamente tratado e utilizado. O framework ainda ressalta a necessidade de plena rastreabilidade de todo o fluxo informacional, desde a sua origem nas fontes do Big Data e IoT até a saída na forma de relatórios e consultas contábeis.

Apesar de ter sido derivado da combinação de modelos existentes na literatura, uma das limitações deste trabalho é que o framework proposto carece de validação. Para tanto, vislumbram-se alguns cenários para sua aplicação. O primeiro consiste em ter a área de TI para validar a aplicação do framework e das categorias de Governança da Informação (Qualidade, Tempestividade e Segurança) nos fluxos de entrada derivados do Big Data e IoT. Um segundo cenário compreende ter os usuários contábeis como base para testes, controles e validação da dinâmica de Governança da Informação. Do ponto de vista empírico, vislumbra-se como etapa futura desta pesquisa a aplicação deste framework na área de Controladoria em uma empresa nacional do setor siderúrgico que vem utilizando o sistema ERP SAP há cerca de 25 anos e que está passando por transformações da Indústria 4.0 em sua operação.

Como limitador de pesquisa, tem-se que considerar que a estrutura de Governança da Informação deve ser desenhada considerando também as características de cada organização (Weber, Otto, \& Österle, 2009), e que ajustes podem ser necessários para a customização do framework em cada contexto. Observa-se, ainda, a existência de outros módulos ERP para além dos contábeis, os quais não foram analisados nesta pesquisa.

Entende-se que uma das contribuições desta pesquisa reside na oferta de um framewok para a estrutura da Governança de Informação necessária para lidar com os recursos trazidos pela Indústria 4.0 no Sistema ERP, despertando o interesse de auditores, contadores, profissionais de TI, gestores e usuários da informação. O uso de um framework baseado em pilares de qualidade, tempestividade e segurança da informação permite uma avaliação contínua de lacunas que representam riscos, contribuindo para a avaliação periódica da maturidade, bem como a melhoria contínua dos controles internos por meio da Governança da Informação. 


\section{REFERÊNCIAS}

Akerkar, R. (2014). Big Data computing. Florida, USA: CRC Press, Taylor \& Francis Group.

Andrade, A., \& Rossetti, J. P. (2009). Governança corporativa: fundamentos, desenvolvimento e tendências (3a. ed.). São Paulo: Atlas.

Araújo, M. A. d., Lima, T. L. d. A., \& Sobral, M. F. F. (2018). Identificando as inovações por meio da adoção dos sistemas erp pelas usinas sucroalcooleiras em pernambuco. Revista Exacta, 16(2), 119-133. doi: 10.5585/exactaep.v16n2.7131

Atkinson, A. A., Kaplan, R. S., Matsumura, E. M., \& Young, S. M. (2011). Management accounting: information for decision-making and strategy execution, student value edition (6a. ed.). EUA: Pearson.

Beijer, P., Kooper, M., \& Advisory, K. (2010). Information governance: beyond risk and compliance. In Proceedings of the european conference on management, leadership 6 governance (p. 28-29).

Blair, B., \& Murphy, B. (2011). Defining information governance: Theory or action? results of the 2011 information governance survey. eDiscovery Journal.

Bouwens, J., \& Abernethy, M. A. (2000, abr. ). The consequences of customization on management accounting system design. Accounting, Organizations and Society, 25(3), 221-241. Recuperado de https://doi.org/10.1016/ s0361-3682(99)00043-4 doi: 10.1016/s0361-3682(99)000434

Bruening, P. J., \& Waterman, K. K. (2010, set. ). Data tagging for new information governance models. IEEE Security E Privacy Magazine, 8(5), 64-68. Recuperado de https:/ doi.org/10.1109/msp.2010.147 doi: 10.1109/msp.2010.147

Carraro, W., Meneses, R., \& Brito, C. (2019, out. ). Combining Categories of Management Control Tools for High Performance of Start-ups. Review of Business Management, 21(4), 861-868. Recuperado de https://doi.org/10.7819/ rbgn.v21i5.4022 doi: 10.7819/rbgn.v21i5.4022

Coyne, E. M., Coyne, J. G., \& Walker, K. B. (2018, mar. ). Big Data information governance by accountants. International Journal of Accounting $\mathcal{G}$ Information Management, 26(1), 153-170. Recuperado de https://doi.org/10.1108/ ijaim-01-2017-0006 doi: 10.1108/ijaim-01-2017-0006

Dani, A. C., \& Beuren, I. M. (2018). Mudanças na Controladoria com o Processo de Convergência às Normas Internacionais de Contabilidade. Contabilidad y $\mathrm{Ne}$ gocios, 13(25), 20-41. Recuperado de https://doi.org/ 10.18800/contabilidad.201801.002 doi: 10.18800/contabilidad.201801.002

Dias, R. (2002). Métricas para Avaliação de Sistemas de Informação. Revista Eletrônica de Sistemas de Informação, 1(1). doi: 10.21529/resi.2002.0101002

Dinsmore, P., \& Rocha, L. (2015). Governança corporativa de projetos. Rio de Janeiro: Brasport.

Dutra, R. S., Parente, P. H. N., Dutra, G. B. M., \& Parente, L. O. d. S. S. (2018). Governança Corporativa e Desempenho Empresarial: uma análise sob o enfoque da reputação corporativa das empresas brasileiras. Revista de Auditoria Governança e Contabilidade - RAGC, 6(26), 47-63. Recuperado de http://www.fucamp.edu.br/editora/index.php/ ragc/article/view/1393

Echer, I. C. (2001). A revisão de literatura na construção do trabalho científico. Revista gaúcha de enfermagem, 22(2), $5-20$.

Faria, F. d. A., Maçada, A. C. G., \& Kumar, K. (2012). Governança da Informação: o exemplo do sistema de Pagamentos Brasileiro. In Encontro da anpad (v. XXXVI). Rio de Janeiro, RJ, Brasil: ANPAD. Recuperado de http://siga.arquivonacional.gov.br/images/ siga_novo/SEMINARIOS/VI_SEMINARIO_SIGA/

APRESENTACOES/01-LARISTSA---Programā-de-Gesto -de-Documentos.pdf

Freitas, P. A. d., Reis, E. A. d., Michel, W. S., Gronovicz, M. E., \& de Macedo Rodrigues, M. A. (2013). Information governance, big data and data quality. In 2013 ieee 16th international conference on computational science and engineering (p. 1142-1143).

Furlan, P. K., \& Laurindo, F. J. B. (2019, jun. ). Revisão Bibliográfica Sobre os Fatores Viabilizadores Para a Adoção das Governanças de Dados ou de Informação. GEPROS Gestão da Produção Operações e Sistemas, 14(2). Recuperado de https://doi.org/10.15675/gepros.v14i2.2217 doi: 10.15675 /gepros.v14i2.2217

Gianella, S., \& Gujer, W. (2006). Improving the information governance of public utilities through an organizational knowledge base. In J. Mathew, K. J., L. Ma, A. Tan, \& D. Anderson (Eds.), Engineering asset management (p. 406417). London: Springer.

Gärtner, B., \& Hiebl, M. R. W. (2018). Issues with Big Data. In M. Quinn \& E. Strauss (Eds.), The Routledge Companion to Accounting Information Systems (p. 161-172). London: Routledge.

Haes, S. D., \& Van Grembergen, W. (2009). An exploratory study into IT governance implementations and its impact on business/IT alignment. Information Systems Management, 26 (2), 123-137. Recuperado de https://doi.org/10.1080/ 10580530902794786 doi: 10.1080/10580530902794786

Hasan, M. S., Ebrahim, Z., Mahmood, W. H. W., \& Rahman, M. N. A. (2018). Factors influencing enterprise resource planning system: A review. Journal of Advanced Manufacturing Technology, 12(1), 247-258. Recuperado de https://jamt.utem.edu.my/jamt/article/view/3939

Hashem, I. A. T., Yaqoob, I., Anuar, N. B., Mokhtar, S., Gani, A., \& Khan, S. U. (2015). The rise of "big data" on cloud computing: Review and open research issues. Cognitive Computing e Big Data Analytics, 47, 98-115. doi: 10.1016/j.is.2014.07.006

Hurwitz, J., Kaufman, M., \& Bowles, A. (2015). Cognitive Computing e Big Data Analytics (v. 1). Wiley Publishing.

International Business Machines [IBM]. (2019). What is Industry 4.0? Recuperado de https://www.ibm.com/br-pt/ services/process/iot-consulting

Kanagaretnam, K., Lobo, G. J., \& Whalen, D. J. (2007). Does good corporate governance reduce information asymmetry around quarterly earnings announcements? Journal of Accounting and Public Policy, 26(4), 497-522. Recuperado de http://www.sciencedirect.com/science/article/pii/ S0278-4254(07)00039-7

Khatri, V., \& Brown, C. V. (2010). Designing data governance. Communications of the ACM, 53(1), 148-152. doi: $10.1145 / 1629175.1629210$

Kooper, M. N., Maes, R., \& Lindgreen, E. R. (2011). On the governance of information: Introducing a new concept of governance to support the management of information. International journal of information management, 31(3), 


\section{5-200. doi: 10.1016/j.ijinfomgt.2010.05.009}

Lazzarotto, M. (2015). Governança corporativa: uma exploração de informações. Revista UNEMAT de Contabilidade, 4(7), 90-102. Recuperado de https://periodicos.unemat.br/ index.php/ruc/article/view/314

Li, Q., Lan, L., Zeng, N., You, L., Yin, J., Zhou, X., \& Meng, Q. (2019). A framework for big data governance to advance RHINs: A case study of china. IEEE Access, 7, 50330-50338. Recuperado de https://doi.org/10.1109/access.2019.2910838 doi: 10.1109/access.2019.2910838

Maçada, A. C. G., Brinkhues, R. A., Freitas Jr, J. C. D. S., \& Lajara, T. T. (2019). Princípios e fatores motivadores para adoção das governanças corporativa de ti e da informação. Revista Alcance, 26(2), 131-147. doi: 10.14210/alcance.v26n2(Mai/Ago).p131-147

Mangueira, L. G., \& Alves, A. S. (2014). Análise comparativa dos principais modelos de maturidade destinados à gestão de dados. In $X$ congresso nacional de excelência em gestão, rio de janeiro.

Medeiros, A. P. d., Lavarda, R. A. B., \& Erdmann, R. H. (2020). Strategy materiality and technology implementation practices in "Industry 4.0" context. Revista Gestão \& Tecnologia, 20(2), 279-301. Recuperado de http://www.spell.org.br/documentos/ver/ 58034/materialidade-da-estrategia-e-a-implementacao-de -tecnologia-na-pratica-no-contexto-da--industria-4-0--/i/ pt-br

Meirelles, T. (2005). A Governança corporativa, análise do desempenho financeiro e a geração de valor aos acionistas: um estudo das Companhias de Capital Aberto que aderiram as práticas de Governança Corporativa [Trabalho de conclusão de curso]. Departamento de Ciências da Administração, Universidade Federal de Santa Catarina - UFSC. Recuperado de http://tcc.bu.ufsc.br/Adm294838.PDF

Melkas, H., \& Uotila, T. (1999). Quality of data, information and knowledge in technology foresight processes. Semantic Scholar. Recuperado de http://mitiq.mit.edu/iciq/ PDF/QUALITY\%20OF\%20DATA,\%20INFORMATION\% 20AND\%20KNOWLEDGE\%20IN\%20TECHNOLOGY\% 20FORESIGHT\%20PROCESSES.pdf

Morais, M. d. O., Moura, I. d., \& Denani, A. L. (2018). A integração entre conhecimento, inovação e indústria 4.0 nas organizações. Brazilian Journal of Development, 4(6), 37163731. Recuperado de https://www.brazilianjournals.com/ index.php/BRJD/article/view/359 doi: 10.34117/bjdv4n7359

Neupane, N. (2020, dez. ). Conceptualizing the pathways of literature review in research. Journal of Practical Studies in Education, 2(1), 1-7. Recuperado de https://doi.org/ 10.46809/jpse.v2i1.16 doi: 10.46809/jpse.v2i1.16

Pinto, V. A., Cardoso, A. M. P., Pinheiro, M. M. K., \& Parreiras, F. S. (2019). Iinterdisciplinarity in data science over big data: findings for mining industry. Inf. \& Soc. Est., 29(4), 61-74. Recuperado de https://www.proquest.com/docview/2417890386 ?pq-origsite $=$ gscholar $\&$ fromopenview $=$ true

Presser, N. H., \& da Silva, E. L. (2019, out. ). Framework da qualidade da informação. Navus - Revista de Gestão e Tecnologia, 9(4), 06-09. Recuperado de https://doi.org/10.22279/navus.2019.v9n4.p06-09.1081 doi: 10.22279/navus.2019.v9n4.p06-09.1081

Proença, D., Vieira, R., \& Borbinha, J. (2016). A maturity model for information governance. In 11th iberian confe- rence on information systems and technologies (cisti). Las Palmas. Recuperado de https://www.inesc-id.pt/ficheiros/ publicacoes/9849.pdf

Rigon, E. A., \& Westphall, C. M. (2013, abr. ). Modelo de Avaliação da Maturidade da Segurança da Informação. Revista Eletrônica de Sistemas de Informação, 12(01), 3. Recuperado de https://doi.org/10.5329/resi.2013.1201003 doi: 10.5329/resi.2013.1201003

Santos, B. P., Alberto, A., Lima, T. D. F. M., \& CharruaSantos, F. M. B. (2018). Indústria 4.0: desafios e oportunidades. Revista Produção e Desenvolvimento, 4(1), 111-124. doi: $10.32358 / \mathrm{rpd}$.2018.v4.316

Santos, S. (2018). Introdução à indústria 4.0: saiba tudo sobre a revolução das máquinas (v. 1). São Paulo: Editora Independently Published.

Schwab, K. (2019). A quarta revolução industrial. São Paulo: Edipro.

Sharma, K. (2017). Visão de processo industrial automation. Elsevier Science.

Shkurskii, S. A., \& Sabel'nikova, E. A. (2011). Resourcecontrol system based on the sap erp system. Coke and Chemistry, 54(11), 426-427.

Simões, S. A., Rodrigues, L. C., Maccari, E. A., \& Pereira, M. F. (2011, dez. ). Managing IT as a business: The lutchen's gap in the 100 top organizations based in brazil. JISTEM Journal of Information Systems and Technology Management, 8(3), 717-748. Recuperado de https://doi .org/10.4301/s1807-17752011000300010 doi: 10.4301/s180717752011000300010

Smallwood, R. F., Kahn, R., \& Murphy, B. (2014). Information governance: Concepts, strategies and best practices. Hoboken: John Wiley \& Sons.

Souza, A. B., Bauer, M. M., \& Coletti, L. (2020, jan. ). A importância da governança corporativa e do controle interno na área contábil. Revista Gestão e Desenvolvimento, 17(1), 148-174. Recuperado de https://doi.org/10.25112/ rgd.v17i1.1723 doi: 10.25112/rgd.v17i1.1723

Telukdarie, A., Buhulaiga, E., Bag, S., Gupta, S., \& Luo, Z. (2018). Industry 4.0 implementation for multinationals. Process Safety and Environmental Protection, 118, 316-329. Recuperado de https://doi.org/10.1016/j.psep.2018.06.030 doi: $10.1016 /$ j.psep.2018.06.030

Teodoro, A. N., Przeybilovicz, É., \& Cunha, M. A. (2014). Governança de tecnologia da informação: uma investigação sobre a representação do conceito. Revista de Administração, 49(2), 307-321. Recuperado de https://doi.org/10.5700/ rausp1148 doi: 10.5700/rausp1148

Trindade, A. L. B., Oliveira, M., \& Becker, G. V. (2011). Análise dos atributos para avaliação da qualidade da informação nos ambientes de intranet para apoio à gestão do conhecimento. REAd. Revista Eletrônica de Administração (Porto Alegre), 17, 776-801. Recuperado de https://seer.ufrgs.br/read/article/view/38523

Weber, K., Otto, B., \& Österle, H. (2009, jun. ). One size does not fit all-a contingency approach to data governance. Journal of Data and Information Quality, 1(1), 1-27. Recuperado de https://doi.org/10.1145/1515693.1515696 doi: $10.1145 / 1515693.1515696$

Zonnenshain, A., \& Kenett, R. S. (2020, fev. ). Quality 4.0- the challenging future of quality engineering. Quality Engineering, 32(4), 614-626. Recuperado de https://doi.org/10.1080/08982112.2019.1706744 doi: 
$10.1080 / 08982112.2019 .1706744$

Como citar este artigo (APA):

Silva, M. D. T., Carvalho, R. B., Castro, J. M. de \& Soares, M. V. (2021). Governança da informação no contexto dos módulos contábeis dos sistemas ERP para a Indústria 4.0: Proposta de framework. AtoZ: novas práticas em informação e conhecimento, 10(3), 1 - 10. Recuperado de: http://dx.doi.org/10.5380/ atoz.v10i3.81477 